\title{
Inequalities and outcomes: end stage kidney disease in ethnic minorities
}

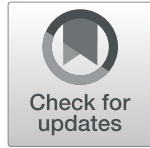

Emma Wilkinson ${ }^{1}$, Alison Brettle ${ }^{2}$, Muhammad Wagar ${ }^{1}$ and Gurch Randhawa ${ }^{*^{*}}$ (D)

\begin{abstract}
Background: The international evidence about outcomes of End Stage Kidney Disease (ESKD) for ethnic minorities was reviewed to identify gaps and make recommendations for researchers and policy makers.

Methods: Nine databases were searched systematically with 112 studies from 14 different countries included and analysed to produce a thematic map of the literature.

Results: Reviews $(n=26)$ highlighted different mortality rates and specific causes between ethnic groups and by stage of kidney disease associated with individual, genetic, social and environmental factors. Primary studies focussing on uptake of treatment modalities $(n=19)$ found ethnic differences in access. Research evaluating intermediate outcomes and quality of care in different treatment phases $(n=35)$ e.g. dialysis adequacy, transplant evaluation and immunosuppression showed ethnic minorities were disadvantaged. This is despite a survival paradox for some ethnic minorities on dialysis seen in studies of longer term outcomes $(n=29)$ e.g. in survival time post-transplant and mortality. There were few studies which focussed on end of life care $(n=3)$ and ethnicity. Gaps identified were: limited evidence from all stages of the ESKD pathway, particularly end of life care; a lack of system oriented studies with a reliance on national routine datasets which are limited in scope; a dearth of qualitative studies; and a lack studies from many countries with limited cross country comparison and learning.

Conclusions: Differences between ethnic groups occur at various points and in a variety of outcomes throughout the kidney care system. The combination of individual factors and system related variables affect ethnic groups differently indicating a need for culturally intelligent policy informed by research to prevent disadvantage.
\end{abstract}

Keywords: Inequalities, Ethnic minorities, Kidney disease, Scoping, Review

\section{Background}

Inequalities in some aspects of End Stage Kidney Disease (ESKD) in the United Kingdom (UK) had been well documented prior to this study. However, to ensure that future research would be based on the most comprehensive evidence this scoping review was commissioned by a national kidney charity in 2017, the topic having been identified through prioritisation process involving people affected by kidney disease as well as clinicians and researchers. This review was the first step in a systematic approach to learn as much as possible from the evidence about inequalities in outcomes for people living and dying with ESKD who are from ethnic minority groups. This paper describes the approach (protocol) used, presents a map of the literature

\footnotetext{
* Correspondence: gurch.randhawa@beds.ac.uk; Gurch.randhawa@beds.ac.uk ${ }^{1}$ Institute for Health Research, University of Bedfordshire, Luton, Bedfordshire, UK

Full list of author information is available at the end of the article
}

and highlights where there are gaps to be addressed through future research and policy.

Investigating inequalities in kidney care and how they impact upon ethnic minorities is not only necessary for reducing inequalities in the UK but is also likely to benefit the understanding of access to care for other groups with ESKD globally as well. This review therefore took a broad perspective and incorporated the international literature. The objectives of this international systematic scoping review were to:

- identify the evidence base of inequalities in the outcomes of end stage kidney disease for ethnic minorities;

- map the international literature;

- identify gaps and make recommendations for research and policy towards reducing and

(C) The Author(s). 2019 Open Access This article is distributed under the terms of the Creative Commons Attribution 4.0 International License (http://creativecommons.org/licenses/by/4.0/), which permits unrestricted use, distribution, and reproduction in any medium, provided you give appropriate credit to the original author(s) and the source, provide a link to the Creative Commons license, and indicate if changes were made. The Creative Commons Public Domain Dedication waiver (http://creativecommons.org/publicdomain/zero/1.0/) applies to the data made available in this article, unless otherwise stated. 
preventing inequalities in kidney care in the UK and globally.

\section{Methods}

This systematic scoping review was conducted in line with the 5 stage framework outlined by Arksey and O'Malley [1]: protocol and search question development; systematic searching and record management; sifting and refining; charting and mapping. The research question was initially determined via a Delphi study and then refined via discussion within the project team to "Why do ethnic minority patients with End Stage Kidney Failure (ESKD) experience poorer outcomes than nonethnic minority patients?".

A systematic scoping review is an early and essential step towards answering the 'why' question but necessarily falls short of doing so as it does not apply or build theory for this. It provides a map of the literature, highlights the issues that other researchers have addressed and shows where there are gaps. It follows a systematic protocol as described in this Methods section and offers up observations and recommendations for next steps which are listed in the Conclusion.

The search strategy and literature searches were formulated and refined by $\mathrm{EW}$ and $\mathrm{AB}$ to be comprehensive and include sources from health, sociology and psychology which considered minority health and any aspect of end stage kidney disease (dialysis, renal replacement therapy, transplant or conservative care and end of life care) in comparison to non-ethnic minorities and in relation to the following outcomes:

- Morbidity, mortality, kidney related, service related, transplant related

- Measures of mental health (e.g. reduction in anxiety or depression using validated tools)

- Measures of emotional well-being or quality of life: (using validated tools).

The definition of ethnic minority was taken from Culley [2] and ethnicity defined as 'a consciousness of belonging to a particular group based on commonality of family origin and culture of shared values and beliefs which is socially constructed'.

A time frame of 1992 onwards was set to capture evidence from the last 25 years. The searches were wide and sensitive and encompassed a range of thesaurus and free text terms to describe the different descriptors of ethnic minorities and the capture the terms related to end stage kidney failure or disease. Table 1 provides an example of one search strategy for one database.

9 electronic databases were searched to identify studies for inclusion in the review (see Table 2 below). There was insufficient resource within the project to search journals and grey literature that could have expanded the potential range of studies to be included.

The searches were undertaken by EW and $\mathrm{AB}$ in $\mathrm{Au}$ gust 2017 and all records were uploaded on to the Endnote database. Using the inclusion and exclusion criteria shown in Table 3, each record was screened independently on the basis of title by the researcher (EW). A $10 \%$ sample of titles were screened by a different member of the research team, any discrepancies resolved, and then the studies were screened by abstract and full text by EW and either included or excluded according to the criteria.

Figure 1 illustrates the searching and sifting process for this review. Data was extracted into a series of evidence tables by EW and MW following project team discussions regarding the column headings and data to be extracted. This included details on author, publication year, author country, ethnic group(s) in focus, research type, research objective, outcomes / indicators and brief summary of findings.

The data was analysed thematically and a map of the data produced to aid analysis, see Fig. 3.

\section{Results}

112 studies were included in this review. These focussed on ESKD and included analysis of outcomes for ethnic minorities in 14 different countries listed in Fig. 2 which shows a breakdown of the included papers by author country of origin and / or country focus. The majority of included studies concerned the US $(n=72)$ followed by the UK $(n=15)$, Australia $(n=9)$, Canada $(n=4)$ and the Netherlands and New Zealand $(n=3)$ with the other eight countries (Singapore, Brazil, Mexico, Romania, Israel, France, Hungary and India) being the focus of only one or two studies each.

Included studies related to five different areas or themes which were identified as: an overview of the question topic area in the form of a review (Theme 1); access to a modality of RRT care i.e. access to haemodialysis, peritoneal dialysis, conservative care (Theme 2); care on dialysis and / or being listed for a transplant and moving through the transplant process with a focus on intermediate outcomes (Theme 3); longer term outcomes and survival (Theme 4) and end of life care (Theme 5).

\section{Theme 1 reviews of the topic area}

Twenty-six included studies were reviews, none of which systematic, and they ranged in scope and detail subdividing into five subsets: those with a focus on CKD, hypertensive kidney disease, a general focus, dialysis and transplantation. 
Table 1 Example of one search strategy (Medline database)

1. exp. Kidney Failure, Chronic/

2. exp. Renal Replacement Therapy/

3. kidney/

4. exp. kidney diseases/

5. exp. renal insufficiency/

6. exp. renal dialysis/

7. Glomerular Filtration Rate/

8. transplantation.mp. [mp = title, abstract, original title, name of substance word, subject heading word, keyword heading word, protocol supplementary concept word, rare disease supplementary concept word, unique identifier, synonyms]

9. 1 or 2 or 3 or 4 or 5 or 6 or 7 or 8

10. Treatment Outcome/ or "Outcome Assessment (Health Care)"/ or outcome assessment.mp. or "Outcome and Process Assessment (Health (are)"I

11. outcome measure.$m p$. [mp = title, abstract, original title, name of substance word, subject heading word, keyword heading word, protocol supplementary concept word, rare disease supplementary concept word, unique identifier, synonyms]

12. exp. Health Status/

13. exp. "Quality of Life"/

14. health impact assessment/

15. well-being.mp.

16. exp. Mental Disorders/

17. exp. Depression/

18. Anxiety/

19. Stress, Psychological/

20. exp. Dementia/

21. exp. Cardiovascular Diseases/

22. end of life care.mp. or Terminal Care/

23. palliative care.mp. [mp = title, abstract, original title, name of substance word, subject heading word, keyword heading word, protocol supplementary concept word, rare disease supplementary concept word, unique identifier, synonyms]

24. conservative care. $\mathrm{mp}$. [ $\mathrm{mp}=$ title, abstract, original title, name of substance word, subject heading word, keyword heading word, protocol supplementary concept word, rare disease supplementary concept word, unique identifier, synonyms]

25. mortality.mp. [ $\mathrm{mp}=$ title, abstract, original title, name of substance word, subject heading word, keyword heading word, protocol supplementary concept word, rare disease supplementary concept word, unique identifier, synonyms]

26. morbidity.mp. [mp = title, abstract, original title, name of substance word, subject heading word, keyword heading word, protocol supplementary concept word, rare disease supplementary concept word, unique identifier, synonyms]

27. survival.mp. [mp = title, abstract, original title, name of substance word, subject heading word, keyword heading word, protocol supplementary concept word, rare disease supplementary concept word, unique identifier, synonyms]

28. 10 or 11 or 12 or 13 or 14 or 15 or 16 or 17 or 18 or 19 or 20 or 21 or 22 or 23 or 24 or 25 or 26 or 27

29. exp. Ethnic Groups/

30. exp. Continental Population Groups/

31. exp. Minority Health/

32. bme.mp.

33. bame.mp.

34. racial.mp.

35. cald.mp. [mp = title, abstract, original title, name of substance word, subject heading word, keyword heading word, protocol supplementary concept word, rare disease supplementary concept word, unique identifier, synonyms]

36. south asian.mp. [mp = title, abstract, original title, name of substance word, subject heading word, keyword heading word, protocol supplementary concept word, rare disease supplementary concept word, unique identifier, synonyms]

37. indo asian.mp. [mp = title, abstract, original title, name of substance word, subject heading word, keyword heading word, protocol supplementary concept word, rare disease supplementary concept word, unique identifier, synonyms]
Table 1 Example of one search strategy (Medline database) (Continued)

38. indian.mp. [mp = title, abstract, original title, name of substance word, subject heading word, keyword heading word, protocol supplementary concept word, rare disease supplementary concept word, unique identifier, synonyms]

39. caucasian.mp. [ $\mathrm{mp}=$ title, abstract, original title, name of substance word, subject heading word, keyword heading word, protocol supplementary concept word, rare disease supplementary concept word, unique identifier, synonyms]

40. exp. African Americans/

41. exp. Hispanic/

42. exp. White/

43. exp. Black/

44. indigenous.mp. [mp = title, abstract, original title, name of substance word, subject heading word, keyword heading word, protocol supplementary concept word, rare disease supplementary concept word, unique identifier, synonyms]

45. inequality.mp. [mp = title, abstract, original title, name of substance word, subject heading word, keyword heading word, protocol supplementary concept word, rare disease supplementary concept word, unique identifier, synonyms]

46. exp. Health Status Disparities/ or exp. Prejudice/ or exp. Ethnic Groups/ or intersectionality.mp. or exp. "Emigration and Immigration"/ or exp. Social Class/

47. 29 or 30 or 31 or 32 or 33 or 34 or 35 or 36 or 37 or 38 or 39 or 40

or 41 or 42 or 43 or 44 or 45 or 46

48. exp. research design/

49. exp. empirical research/

50. exp. qualitative research/

51.48 or 49 or 50

52.9 and 28 and 47 and 51

53. limit 52 to $y r .=" 1992$-Current"

\section{Reviews with a focus on CKD [3-7]}

As survival and risk change over time and according to stage and treatment, these studies suggested that interventions should be stratified and tailored in a similar way with prospective longitudinal and cohort research needed which include ethnic minority groups. Furthermore, the multiplicity of factors which put different ethnic minorities at increased risk of CKD and ESKD (hypertension, Type 2 Diabetes Mellitus (T2DM), albuminuria) call for a complex analysis of system-related as well as physiological factors through more cross population multi-levels studies. The urgency of prevention in countries where there are geographical and economic constraints on access to dialysis but increasing incidence in ESKD amongst particular indigenous groups, highlighted the ethical and moral necessities of intervention from a global perspective.

\section{Review with a focus on hypertensive kidney disease [8]}

This paper illustrated the different patterns in ESKD among different ethnic groups within diverse populations which vary from country to country and how ethnicity relates to hypertensive kidney disease as the predominant cause of ESKD for African Americans. The range of mediators which can impact on hypertensive ESKD e.g.: socio-demographics; access to healthcare; 
Table 2 Databases searched and number of references retrieved

\begin{tabular}{lll}
\hline Database name & Focus & Number of references retrieved \\
\hline Medline & Biomedical & 185 \\
Cinahl & Nursing and allied health & 534 \\
Psychinfo & Psychology/Mental Health & 78 \\
Web of Science/Web of Knowledge & Science/Social Sciences & 224 \\
Scopus & General & 577 \\
Social Care Online & Social Care & 53 \\
ASSIA & Social Science & 370 \\
Global Health & Health & 97 \\
Cochrane Database of Promoting Health Effectiveness Reviews & Systematic Reviews & 17 \\
Total 9 databases & & 2135
\end{tabular}

blood pressure management; and differences in outcomes on renal replacement therapy (RRT) were described. The earlier onset and more severe disease could be partly explained by worse access to healthcare associated with lower socioeconomic status.

\section{Reviews with a general focus [9-11]}

These studies provide some general observations on inequalities in relation to a variety of different ethnic groups and African and Native Americans. There is more rapid progression from CKD to ESKD in ethnic minorities; minority groups have worse access to care and late referral to specialist renal care. Although transplantation is the 'best' treatment for many people, wait time to transplantation is longer for ethnic minorities due to delays in completing the transplant evaluation process and because of lack of suitable matched organs. These three reviews draw attention to the need for culturally competent prevention at the same time as continuing with research to investigate why there are different outcomes seen in ethnic minority groups. The influence of socioeconomic factors such as education, social status and associated attitudes towards treatment options for
ESKD, and quality of life on RRT compared to majority population groups, are aspects which require better understanding.

\section{Reviews with a focus on dialysis [12-16]}

Five reviews focussed on ESKD from the perspective of dialysis services in the UK, Europe, the US and other parts of the world e.g. Israel. The overarching premise was that the incidence of ESKD and associated need for dialysis is steadily increasing with differences between ethnic groups and associated with changing age structure of populations. These reviews with a focus on different aspects of dialysis care came with the caveat that long term survival of ESKD is very low and survival advantages on dialysis are marginal compared to transplantation with a living donor graft which can extend life considerably but still not back to normal lifespan. Although a survival advantage has been seen in some ethnic groups on dialysis a need for a better understanding of the underlying mechanisms behind the differences and taking action on barriers to access both in the pathway to ESKD but also in access to care on dialysis including transplantation was highlighted.

Table 3 Inclusion and Exclusion Criteria

\begin{tabular}{|c|c|}
\hline Inclusion & Exclusion \\
\hline $\begin{array}{l}\text { - Studies (including systematic reviews and all study designs) that mention } \\
\text { ethnic minority health in line with the above definition and in relation to end } \\
\text { stage kidney disease and related descriptions (end stage renal failure, dialysis, } \\
\text { end of life kidney care). } \\
\text { - Evidence of a measurable outcome on health or wellbeing (e.g. physical or } \\
\text { mental health or physiological or quality of life or wellbeing) } \\
\text { - Studies which concern any of the related end stage kidney disease descriptors: } \\
\text { - Patients on any kind of dialysis } \\
\text { - Patients on Renal replacement therapy } \\
\text { o Transplant patients } \\
\text { - End stage renal failure } \\
\text { - Adults over } 18 \\
\text { - Studies in English, post } 1992 \\
\text { - Any country } \\
\text { - A comparison with non-ethnic minority group (s) }\end{array}$ & $\begin{array}{l}\text { - Studies outside the agreed definition of ethnic minority } \\
\text { - Studies with no measurable outcomes that relate to health } \\
\text { - Studies which do not concern any of the related end stage } \\
\text { kidney failure descriptors: } \\
\text { - Patients on any kind of dialysis } \\
\text { - Transplant patients } \\
\text { - End stage renal failure } \\
\text { - Children less than } 18 \\
\text { - Studies in languages other than English, prior to } 1992 \\
\text { - Studies that do not have a comparison with non-ethnic } \\
\text { minority group (s) } \\
\text { - Studies that are commentaries, editorials (which do not } \\
\text { present data), thought pieces or conference abstracts }\end{array}$ \\
\hline
\end{tabular}


Potentially relevant citations identified through searching $\mathrm{n}=2135$ citations

Studies excluded after duplicate removal, title review, reading abstracts and full text

$n=2023$

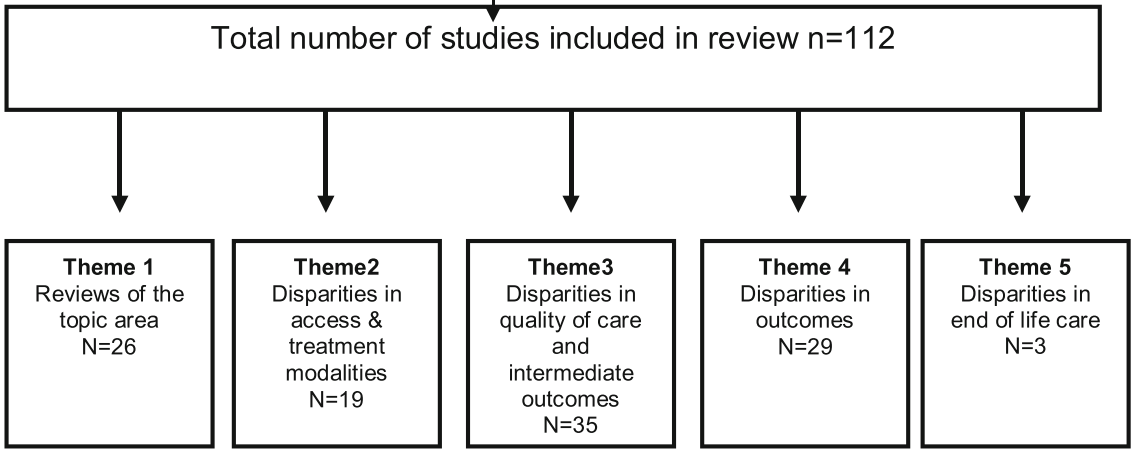

Fig. 1 Searching and sifting process

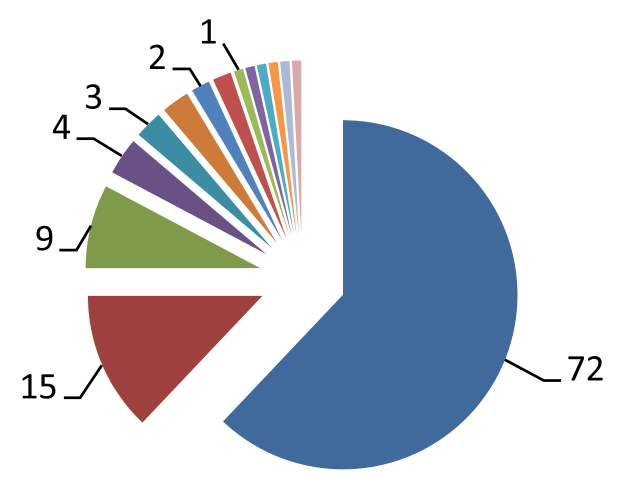

\begin{tabular}{|c|c|c|c|c|}
\hline USA & UK & Australia & - Canada & Netherlands \\
\hline New & Singapore & Brazil & Mexico & Romania \\
\hline Israe & France & Hungary & India & \\
\hline
\end{tabular}

Fig. 2 Breakdown of included articles by country of author origin and / or focus 
Reviews with a focus on transplantation [17-28]

Twelve reviews concerned inequalities in the transplantation process or transplantation outcomes. The overarching premise being that ethnic minorities, although disproportionately represented in patient populations with ESKD, receive fewer transplants and wait longer to receive a transplant. These reviews gave perspectives that varied according to the level of focus: global; country; population; individual as well as stage in the transplant process (pre-emptive transplantation, referral to waiting list, organ allocation, graft survival and immunosuppression) and donation. Overarching conclusions were that better understanding and communication would lead to increased awareness of how the social and individual risk factors interact through the system which is necessary for improving access and reducing inequalities.

\section{Theme 2 disparities in access \& treatment modalities}

Nineteen studies reported access to kidney care treatment modalities - dialysis, transplant and conservative care, post diagnosis of ESKD.

Sixteen out of the 19 were cohort designs, 1 observational - cross sectional, 1 mixed method and ethnographic, 1 other (review of registry report data). The studies spanned 6 countries.

Indicators related to access to RRT modalities were: incidence or prevalence on the modality; rate of acceptance; planned and unplanned initiation; time to modality; waiting list probability; time from acceptance onto waiting list to transplantation (and rate); time from start of RRT to transplantation (and rate); transplantation rate.

\section{Access to dialysis - peritoneal dialysis and hemodialysis [29-37]}

Studies described differences in uptake of dialysis modality by different ethnic minority groups compared to majority populations. These were not consistent between different countries and different minority populations highlighting that despite commonalities ethnic minorities are diverse groups whose experiences vary from country to country and on an individual level.

The sociocultural context of renal replacement therapy featured as an important consideration with a focus on possible explanatory factors for the larger prevalent ethnic minority population on dialysis compared to the general population. A third of the countries represented in this review had a national kidney data service which contributed to the research picture by providing quantitative analysis of some elements.

\section{Access to transplantation [38-47]}

Studies showed that ethnic minorities with ESKD are disproportionately represented in the transplantation modality and identified a range of reasons that relate to the different countries' transplant systems. These included the rates of being listed for transplant; movement from list to transplantation; variations across transplant centres as well in pre-dialysis care and cadaveric and life donation rates, which all showed disparities when ethnic minorities were compared with majority populations. The requirement for future research and service development to take stratified and systemic approaches towards improving access to transplantation to redress these inequalities was raised - as despite being highly technical, dialysis and transplantation are part of a complex process which is influenced by human and sociological factors.

\section{Theme 3 disparities in quality of care and intermediate outcomes}

Thirty-five studies investigated ethnic differences in intermediate outcomes of treatment for ESKD.

Sixteen out of the 35 were cohort designs, 12 other observational (cross sectional, survey, discrete choice experiment); 3 other (programme review, record review, simulation), 3 qualitative and 1 randomised controlled trial (RCT). The studies spanned 6 countries.

Intermediate outcomes related to kidney function were: estimated glomerular filtration rate (eGFR); proteinuria / serum albumin; indicators of quality of care e.g. dialysis dose / dialysis adequacy expressed as $\mathrm{Kt} / \mathrm{V}$, urea reduction ratio (URR), anaemia and mineral management, infections, hospitalisation, communication \& information; indicators of health and wellbeing or behaviour i.e. nutritional status, quality of life (QOL), coping style; indicators for movement through clinical pathways i.e. through the transplant evaluation and allocation process; transplant indicators e.g. antibody matching, immunosuppression; and indicators of organisational or system function e.g. issues of insurance, allocation priorities, centre level variations, system level intervention.

Ethnic minorities are younger at referral to specialist kidney care and as they often face a longer wait for a transplant so that time spent on dialysis or moving through the transplant process is both an opportunity and a risk for outcomes later in the pathway. The choice of dialysis modality and type of vascular access can be associated with ESKD outcomes and researchers investigated these for disparities amongst ethnic groups.

\section{Quality of care - dialysis [48-63]}

Studies reported on indicators of dialysis care such as hemodialytic dose as well as clinical outcomes such as time to first infection episode and found there these 
varied by different ethnic groups and required further explanation. Other indicators of maintaining health whilst on dialysis and associated outcomes such as quality of life similarly suggested a stratified approach to identifying related inequalities was necessary as there are age, gender and ethnicity associations to be understood. Links back to wider system variables again were made.

\section{Quality of care- transition to transplant [64-75]}

Ethnic disparities in the transition from diagnosis of ESKD or dialysis to transplant were investigated in 10 of the included studies. The low transplant rates for ethnic minorities which are seen overall in many countries can be linked to disparities further back in the pathway well as availability of suitable donor organs. Barriers responsible for differences in completion of stages and movement through the transplant process were associated with minority ethnicity and linked to levels of information given, knowledge and communication between patient and physician but also within wider networks.

\section{Quality of care - the system [76-82]}

Disparities in the transplant process were associated with ethnicity for some groups and related to interaction with kidney allocation and transplantation systems and influenced by a wide range of factors such as geography, clinician-patient communication, insurance and variation across centres in the same system. These studies highlighted the complexity of transplantation systems.

\section{Theme 4 disparities in longer term outcomes}

Twenty-nine studies focussed on patient survival outcomes for dialysis and transplantation.

Twenty-five out of the 29 were cohort designs and 4 were other observational studies. Collectively they investigated differences in outcomes across 12 broad ethnic groups, 3 analysing by ethnic subgroups. The studies spanned 8 countries.

Longer term outcomes relating to dialysis and transplantation were: survival time on dialysis; time posttransplant; hospitalisation, infection; allograft rejection; quality of life (QOL); and mortality.

The outcomes for dialysis and transplantation varied across different ethnicities, subgroups and stratification by other variables: age, comorbidities (type 1 diabetes mellitus (T1DM), type 2 diabetes mellitus (T2DM), Lupus Nephritis) and location.

\section{Overarching outcomes of ESKD [83-87]}

Studies that reported the overarching outcomes of ESKD identified inequalities faced by particular populations and subgroups drawing attention to the disproportionate burden of dialysis and mortality attributed to rates and risk of diabetes and kidney complications. Other studies highlighted how ethnic inequalities in outcomes can be linked to area differences within countries and across regions.

\section{Dialysis outcomes [88-101]}

Some ethnic minorities have been found to have longer survival on dialysis than the majority population and this apparent survival paradox is against the background of increased risk, faster progression to ESKD and poorer survival in the general population overall. The dialysis survival advantage was not seen to be consistent across the groups studied with different ethnicities and comorbidities; cultural and genetic differences as well as variation in access and delivery of care accounting for this.

\section{Transplantation outcomes [102-111]}

The reduced likelihood of having a transplant is a disparity many ethnic minority patients face and studies investigated risks as well as survival outcomes. Ethnic group and comorbidities were found to be associated with this with greater disparities seen when live and deceased donation were compared. To understand the impact of ethnicity on transplant survival outcomes research investigated other risks underpinning delayed graft function, acute rejection episodes, expandedcriteria donor, impaired graft function which for some ethnicities was an independent risk. Differential rates of risks and survival between ethnic groups were found in some studies.

\section{Theme 5 disparities in end of life care [112-114]}

Three studies that focussed on disparities in end of life care for people with ESKD were included in this review. These studies all came from one country, the US. Two had RCT designs and 1 was a cohort study.

Ethnic minorities were less likely to withdraw from dialysis before death than the majority population with differences explained by sociocultural rather than medical factors. Research in this area included two studies which attempted to understand and improve end of life care for all kidney patients through culturally sensitive intervention.

\section{Discussion}

ESKD is a multifaceted condition with many clinical and sociological elements and there are multiple stages and timings to consider in achieving the best outcome for individuals living with the condition. Within the research literature individuals are generally aggregated into groups to look for evidence of inequalities and this international scoping review has identified that there are disparities between ethnic and non-ethnic minority populations in all aspects of ESKD progression and management. 
The search strategy for this review was formulated and conducted by an experienced health researcher (EW) and an expert in literature searching and systematic reviewing $(\mathrm{AB})$. It was designed to be as wide as possible within the resource and time constraints for the work. Tests for publication bias were not conducted nor were reference lists mined or grey literature searched, and these are limitations. However, the research question and search strategy were kept intentionally broad, reviews were included, and the search resulted in studies that used a range of different outcome indicators which related to the different clinical and social elements and stages of care for ESKD. The variety of studies and their juxtaposition within the ESKD field enabled a useful overview of the evidence base, notwithstanding any publication bias.

Figure 3 is a visual representation of the main themes in relation to each other and served to highlight some of the gaps. The different themes corresponded to specific elements of the kidney care system - access to treatment modalities, dialysis, transplantation and end of life care - and this review is the first, as far as we are aware, to map the evidence of inequalities in the ESKD field as a whole. This review therefore is a comprehensive starting point for researchers and policy makers to consider where and in what form evidence exists on inequalities in ESKD for ethnic minorities.

Although this review mapped the literature across a schematic ESKD pathway, it highlighted an absence of longitudinal cohort studies (retrospective or prospective) which followed CKD patients as they progress through that pathway. There was also a dearth of studies in two particular areas of ESKD: system-related outcomes and end of life care. The lack of longitudinal and systemrelated studies may be due to the difficulties of conducting complex systems-related research whereas the lack of end of life studies could reflect a lack of recognition of end of life care as distinct element of kidney care, as well as difficulties in defining end of life and barriers to conducting research in that context.

The literature was also predominantly quantitative and so was limited in helping understand the basis of the differences in outcomes between ethnic groups. Although national registries were a valuable source of data, especially regarding records and analyses of outcomes by ethnic group [13], the national routine datasets do not include indicators for all elements of care, nor analysis at an individual level. Cultural aspects of ethnicity such as attitudes towards death and dying come into play at end of life and there are cultural differences between different parts of healthcare systems so that more qualitative research would help understanding of the individual, cultural and social factors which influence the disparities in outcomes in these and other areas of ESKD. Furthermore, more qualitative and mixed method studies would help to build theories about why inequalities exist and how they can be addressed going forward.

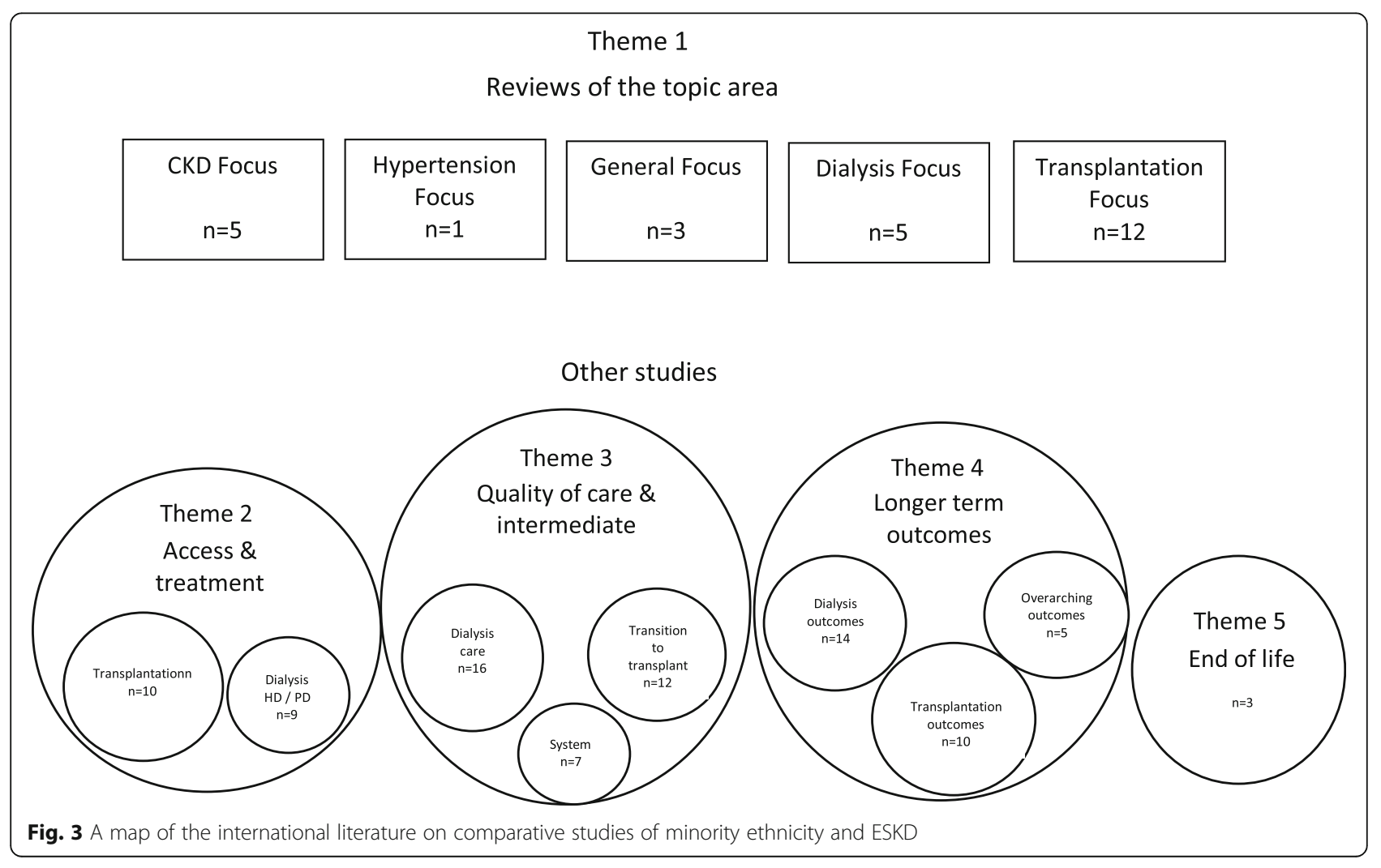


The majority of the evidence for this review has come from a small number of countries and regions notably the US, UK and Australasia. Reasons for the lack of research in other countries, particularly in lower income settings, may reflect how kidney care is funded and delivered in individual countries; the absence of national data sets concerning ESKD; or the lack of ethnicity data. This review highlights the complexities of the kidney and transplant care systems which people with ESKD engage with. Systems in different countries may share some common features but they may also be country specific so that more research and analyses of inequalities in ESKD globally, with cross country comparisons, and a systems approach, would help to share learning and build on this evidence.

Individual countries like the UK, where there is the National Health Service and a national kidney data registry, can not afford to rest on their laurels because as Feehally [9], Lightstone [12] and Randhawa [27] have been suggesting over the past 20 years, the UK's changing demography towards greater ethnic diversity together with a donation shortage, means that without concerted evidencebased intervention ethnic minorities will continue to experience inequalities in outcomes for ESKD. Furthermore, as ethnic minorities are highly heterogeneous groups, culturally intelligent approaches are needed to understand the barriers and enablers of access within individual country systems and to learn from international comparisons.

\section{Conclusions - gaps in the evidence}

Differences between ethnic groups occur at various points and in a variety of outcomes throughout the ESKD care system. The combination of individual factors and system related variables affect ethnic groups differently indicating a need for culturally intelligent policy informed by research to prevent disadvantage.

This thematic analysis contributes to the international research evidence by mapping the different elements of ESKD research where inequalities for ethnic minorities have been observed. A number of gaps in the international literature have been highlighted which if addressed would strengthen our understanding of why inequalities exist for people from ethnic minority groups who are living and dying with ESKD.

\section{Gap 1}

There is a lack of evidence at all stages in the ESKD pathway and a lack of longitudinal cohort studies (retrospective or prospective) which follow CKD patients as they progress through the pathway.

\section{Gap 2}

The lack of system oriented research into ESKD and a reliance on primary studies using national routine data limits our understanding of how the different elements in the system work together.

\section{Gap 3}

There is a lack of qualitative studies contributing to the evidence base and the literature is predominantly quantitative. This limits a full understanding of the factors that influence disparities in ESKD outcomes.

\section{Gap 4}

There is a lack of evidence from many countries apart from the US, especially low-resource settings, and this limits the possible learning across and between countries.

\section{Abbreviations}

CKD: Chronic kidney disease; eGFR: Estimated glomerular filtration rate; ESKD: End stage kidney disease; Kt $/ \mathrm{N}$ : Dialysis adequacy ( $\mathrm{K}=$ dialyser clearance of urea, $t=$ dialysis time, $V=$ volume of distribution of urea); QOL: Quality of Life; RCT: Randomised controlled trial; RRT: Renal replacement therapy; T1DM: Type 1 diabetes mellitus; T2DM: Type 2 diabetes mellitus: URR: Urea reduction ratio

\section{Acknowledgements \\ Not applicable.}

Authors' contributions

EW and $A B$ formulated and conducted the search strategies. EW and MW extracted data into charting tables. EW conducted the analysis with oversight and advice from $A B$ and GR. EW drafted this paper and all authors read and approved the final manuscript.

Funding

This study was supported with funding from Kidney Research UK and the research topic was initiated following a prioritisation process which the charity funded.

Availability of data and materials

Not applicable as this was a review of existing published literature.

Ethics approval and consent to participate

Not applicable as this was a review of existing published literature.

Consent for publication

Not applicable.

Competing interests

The authors declare that they have no competing interests.

\section{Author details}

${ }^{1}$ Institute for Health Research, University of Bedfordshire, Luton, Bedfordshire, UK. ${ }^{2}$ School of Health and Society, University of Salford, Manchester, UK.

Received: 14 November 2018 Accepted: 6 June 2019

Published online: 26 June 2019

\section{References}

1. Arksey H, O'Malley L. Scoping studies: towards a methodological framework. Int J Soc Res Methodol. 2005;8(1):19-32.

2. L C. Nursing, culture and competence. In: Ethnicity and Nursing Practice, C. L.a.D. S. Basingstoke: Palgrave; 2001. p. 109-27. 
3. Alves TP, Lewis J. Racial differences in chronic kidney disease (CKD) and end-stage renal disease (ESRD) in the United States: a social and economic dilemma. Clin Nephrol. 2010;74(SUPPL.1):S72-7.

4. Nicholas SB, Kalantar-Zadeh K, Norris KC. Racial disparities in kidney disease outcomes. Semin Nephrol. 2013;33(5):409-15.

5. Norris KC, Agodoa LY. Unraveling the racial disparities associated with kidney disease. Kidney Int. 2005;68(3):914-24.

6. Patzer RE, McClellan WM. Influence of race, ethnicity and socioeconomic status on kidney disease. Nat Rev Nephrol. 2012;8(9):533-41.

7. Weil EJ, Nelson RG. Kidney disease among the indigenous peoples of Oceania. Ethnicity \& disease. 2006;16(2 Suppl 2):S2-24-30

8. Agodoa L. Hypertensive kidney disease in African Americans. Nephrology. 2001;6(1):25-31.

9. Feehally J. Ethnicity and renal replacement therapy. Blood Purif. 2010;29(2):125-9.

10. Gadegbeku C, Freeman M, Agodoa L. Racial disparities in renal replacement therapy. J Natl Med Assoc. 2002;94(8):45S-54S.

11. Narva, A.S., N.R. Burrows, and S.D. Woodruff, Longer survival among native Americans than among whites who initiated therapy for diabetes-related end-stage renal disease, United States, 1990-2001. J Am Soc Nephrol, 2003. 14: p. 504A-504A.

12. Lightstone L. Preventing renal disease: the ethnic challenge in the United Kingdom. Kidney Int. 2003;63(Supplement 83):S135-8.

13. van den Beukel TO, et al. Racial minority groups on dialysis in Europe: a literature review. Clin Nephrol. 2010;74(SUPPL 1):S78-84.

14. Kalantar-Zadeh $\mathrm{K}$, et al. Survival disparities within American and Israeli dialysis populations: learning from similarities and distinctions across race and ethnicity. Semin Dial. 2010;23(6):586-94.

15. Agodoa L, Eggers P. Racial and ethnic disparities in end-stage kidney failure - survival paradoxes in African-Americans. Semin Dial. 2007;20(6): 577-85.

16. Leggat JE Jr, Swartz RD, Port FK. Withdrawal from dialysis: a review with an emphasis on the black experience. Adv Ren Replace Ther. 1997:4(1):22-9.

17. Callender $\mathrm{CO}$, et al. Blacks and whites and kidney transplantation: a disparity! But why and why won't it go away? Transplant Rev. 2002;16(3):163-76.

18. Young $C$, Kew C. Health disparities in transplantation: focus on the complexity and challenge of renal transplantation in African Americans. Med Clin N Am. 2005;89(5):1003.

19. Garcia GG, Harden P, Chapman J. The global role of kidney transplantation. Curr Opin Nephrol Hypertens. 2012;21(3):229-34.

20. Ghahramani N. Disparities in care of patients with end-stage renal disease: Review of literature and action plan. Iran J Kidney Dis. 2007;1(2):47-53.

21. Vamos EP, Novak M, Mucsi I. Non-medical factors influencing access to renal transplantation. Int Urol Nephrol. 2009;41(3):607-16.

22. Gaston RS, et al. Racial equity in renal-transplantation - the disparate impact of HLA based allocation. Jama-Journal of the American Medical Association. 1993;270(11):1352-6.

23. Joshi S, Gaynor JJ, Ciancio G. Review of ethnic disparities in access to renal transplantation. Clin Transpl. 2012;26(4):E337-43.

24. Wolfe W. Achieving equity in referrals for renal transplant evaluations with afro-American patients: the role of nephrology social workers. Soc Work Health Care. 2003;37(3):75-87

25. Wolfe WA. Increasing African-American living kidney donors: the feasibility and potential role of nephrology nurses and social workers. Soc Work Health Care. 2003;37(4):73-89.

26. Malat $\mathrm{GE}$, et al. African American kidney transplantation survival: the ability of immunosuppression to balance the inherent pre- and post-transplant risk factors. Drugs. 2009;69(15):2045-62.

27. Randhawa G. The impending kidney transplant crisis for the Asian population in the UK. Public Health. 1998;112(4):265-8.

28. Stephens D. Exploring pathways to improve indigenous organ donation. Intern Med J. 2007;37(10):713-6.

29. Barkercummings $C$, et al. Ethnic-differences in the use of peritoneal-dialysis as INITIAL treatment for end-stage renal-disease. Jama-Journal of the American Medical Association. 1995;274(23):1858-62

30. Xue. Peritoneal and hemodialysis: I. Differences in patient characteristics at initiation; 2002.

31. Mehrotra R, et al. Racial and ethnic disparities in use of and outcomes with home Dialysis in the United States. J Am Soc Nephrol. 2016;27(7): 2123-34.

32. Khanal $\mathrm{N}$, et al. Overview of dialysis in indigenous compared to nonindigenous Australians. Clinical Nephrology. 2016;86(13):123-7.
33. McKercher $\mathrm{C}$, et al. Gender differences in the dialysis treatment of indigenous and non-indigenous Australians. Australian \& New Zealand Journal of Public Health. 2017;41(1):15-20.

34. Hemmelgarn BR, et al. Differences in use of peritoneal dialysis and survival among east Asian, indo Asian, and white ESRD patients in Canada. Am J Kidney Dis. 2006;48(6):964-71.

35. Teo BW, et al. Profile of hospitalisation and death in the first year after diagnosis of end-stage renal disease in a multi-ethnic Asian population. Ann Acad Med Singap. 2010;39(2):79-87.

36. Trehan A, et al. End-stage renal disease in indo-Asians in the north-West of England. Qjm-an International Journal of Medicine. 2003;96(7):499-504.

37. RDS U. Incidence, prevalence, patient characteristics, \& treatment modalities. Am J Kidney Dis. 2014;63(1 SUPPL):e215-28.

38. Pruthi $R$, et al. UK renal registry 16th annual report: chapter 4 demography of patients wait-listed for renal transplantation in the UK: national and Centre-specific analyses. Nephron Clinical Practice. 2013;125(1-4):81-98.

39. Collins JF. Kidney disease in Maori and Pacific people in New Zealand. Clin Nephrol. 2010;74:S61-5.

40. Cass A, et al. Renal transplantation for indigenous Australians: identifying the barriers to equitable access. Ethnicity \& Health. 2003;8(2):111-9.

41. Jeffrey RF, et al. Indo-Asian experience of renal transplantation in Yorkshire results of a 10-year survey. Transplantation. 2002;73(10):1652-7.

42. Pruthi $R$, et al. UK renal registry 15th annual report: chapter 9 Centre variation in access to renal transplantation in the UK (2006-2008). Nephron Clinical Practice. 2013;123:183-93.

43. Garg PP, Diener-West M, Powe NR. Reducing racial disparities in transplant activation: whom should we target? Am J Kidney Dis. 2001;37(5):921-31.

44. Purnell TS, et al. Racial differences in determinants of live donor kidney transplantation in the United States. Am J Transplant. 2013;13(6):1557-65.

45. Mohandas $\mathrm{R}$, et al. Racial and socioeconomic disparities in the allocation of expanded criteria donor kidneys. Clinical Journal of The American Society of Nephrology: CJASN. 2013:8(12):2158-64.

46. Gordon EJ. Patients' decisions for treatment of end-stage renal disease and their implications for access to transplantation. Soc Sci Med. 2001;53(8):97187

47. Wolfe RA, et al. Differences in access to cadaveric renal transplantation in the United States. Am J Kidney Dis. 2000;36(5):1025-33.

48. Patibandla BK, et al. Disparities in arteriovenous fistula placement in older hemodialysis patients. Hemodial Int. 2014;18(1):118-26.

49. Woo K, et al. Variations in outcomes of hemodialysis vascular access by race/ethnicity in the elderly. J Vasc Surg. 2017;65(3):783-792.e4.

50. Zarkowsky DS, et al. Racial/ethnic disparities associated with Initial hemodialysis access. JAMA Surgery. 2015;150(6):529-36.

51. Frankenfield DL, et al. Racial/ethnic analysis of selected intermediate outcomes for hemodialysis patients: results from the 1997 ESRD core indicators project. Am J Kidney Dis. 1999:34(4):721-30.

52. Frankenfield DL, et al. Survival advantage for adult Hispanic hemodialysis patients? Findings from the end-stage renal disease clinical performance measures project. J Am Soc Nephrol. 2003;14(1):180-6.

53. Rocco MV, et al. Intermediate outcomes by race and ethnicity in peritoneal dialysis patients: results from the 1997 ESRD Core indicators project. Perit Dial Int. 2000;20(3):328-35.

54. Marley JV, et al. Peritoneal dialysis outcomes of indigenous Australian patients of remote Kimberley origin. Aust J Rural Health. 2014;22(3) $101-8$

55. Yan $\mathrm{G}$, et al. Race/ethnicity, age, and risk of hospital admission and length of stay during the first year of maintenance hemodialysis. Clinical Journal of The American Society of Nephrology: CJASN. 2014;9(8):1402-9.

56. Beavers, Effect of Over-the-Counter Fish-Oil Administration on Plasma Lp(a) Levels in an End-Stage Renal Disease Population. 2009.

57. Rock $\mathrm{CL}$, et al. Racial group differences in plasma concentrations of antioxidant vitamins and carotenoids in hemodialysis patients. Am J Clin Nutr. 1997;65(3):844-50.

58. Bakewell A, Higgins R, Edmunds M. Nutrition, adequacy of dialysis, and clinical outcome in indo-Asian and white European patients on peritoneal dialysis. Qjm-an International Journal of Medicine. 2002;95(12):811-20.

59. Umeukeje EM, et al. Health care providers' support of patients' autonomy, phosphate medication adherence, race and gender in end stage renal disease. J Behav Med. 2016;39(6):1104-14.

60. Yang $F$, et al. Health-related quality of life of Asian patients with end-stage renal disease (ESRD) in Singapore. Qual Life Res. 2015;24(9):2163-71. 
61. Bakewell AB, Higgins RM, Edmunds ME. Quality of life in peritoneal dialysis patients: decline over time and association with clinical outcomes. Kidney Int. 2002;61(1):239-48.

62. Bakewell $A B$, Higgins RM, Edmunds ME. Does ethnicity influence perceived quality of life of patients on dialysis and following renal transplant? Nephrology Dialysis Transplantation. 2001;16(7):1395-401.

63. Greco P, Brickman AL, Routh DK. Depression and coping in candidates for kidney transplantation: racial and ethnic differences. J Clin Psychol Med Settings. 1996;3(4):337-53.

64. Akolekar D, Forsythe JLR, Oniscu GC. Impact of patient characteristics and comorbidity profile on activation of patients on the kidney transplantation waiting list. Transplant Proc. 2013;45(6):2115-22.

65. Keith $\mathrm{D}$, et al. Insurance type and minority status associated with large disparities in prelisting dialysis among candidates for kidney transplantation. Clin J Am Soc Nephrol. 2008;3(2):463-70.

66. Sawinski, Factors associated with failure to list HIV-positive kidney transplant candidates. 2009.

67. Lockwood MB, et al. Patient-reported barriers to the Prekidney transplant evaluation in an at-risk population in the United States. Prog Transplant. 2017;27(2):131-8.

68. Monson RS, et al. Disparities in completion rates of the medical prerenal transplant evaluation by race or ethnicity and gender. Transplantation. 2015; 99(1):236-42.

69. Alexander GC, Sehgal AR. Barriers to cadaveric renal transplantation among blacks, women, and the poor. JAMA. 1998;280(13):1148-52

70. Ismail SY, et al. Modifiable factors in access to living-donor kidney transplantation among diverse populations. Transplantation. 2013;96(6): 586-90.

71. Browne T. The relationship between social networks and pathways to kidney transplant parity: evidence from black Americans in Chicago. Soc Sci Med. 2011;73(5):663-7.

72. Daw J. Of kin and kidneys: do kinship networks contribute to racial disparities in living donor kidney transplantation? Soc Sci Med. 2014; 104:42-7.

73. Ismail SY, et al. Living donor kidney transplantation among ethnic minorities in the Netherlands: a model for breaking the hurdles. Patient Educ Couns. 2013;90(1):118-24

74. Ayanian JZ, et al. Physicians' beliefs about racial differences in referral for renal transplantation. Am J Kidney Dis. 2004;43(2):350-7.

75. Cass. Decision-making about suitability for kidney transplantation - results of a national survey of Australian nephrologists; 2007

76. Reddan DN, et al. Racial inequity in America's ESRD program. Semin Dial. 2000;13(6):399-403.

77. Clark MD, et al. Prioritising patients for renal transplantation? Analysis of patient preferences for kidney allocation according to ethnicity and gender. Diversity in Health \& Care. 2009;6(3):181-91.

78. Anderson K, et al. They really want to go back home, they hate it here: the importance of place in Canadian health professionals' views on the barriers facing aboriginal patients accessing kidney transplants. Health \& Place. 2009; 15(1):390-3.

79. Daumit $\mathrm{GL}$, et al. Use of cardiovascular procedures among black persons and white persons: a 7-year nationwide study in patients with renal disease. Ann Intern Med. 1999;130(3):173.

80. Pallet $\mathrm{N}$, et al. Kidney transplant in black recipients: are African Europeans different from African Americans? Am J Transplant. 2005;5(11):2682-7.

81. Hall EC, et al. Center-level factors and racial disparities in living donor kidney transplantation. Am J Kidney Dis. 2012;59(6):849-57.

82. Vranic GM, Ma JZ, Keith DS. The role of minority geographic distribution in waiting time for deceased donor kidney transplantation. Am J Transplant. 2014;14(11):2526-34

83. Gadalean F, et al. The survival of Roma minority patients on chronic hemodialysis therapy - a Romanian multicenter survey. PLoS One. 2016; 11(5):e0155271.

84. Dyck RF, et al. End stage renal disease among people with diabetes: a comparison of first nations people and other Saskatchewan residents from 1981 to 2005. Can J Diabetes. 2010;34(4):324-33.

85. Abidi SM, et al. Diabetic end-stage renal disease in the indigenous population of the Commonwealth of the Northern Mariana Islands. Nephrology. 2005;10(3):291-5.

86. Boan $\mathrm{P}$, Swaminathan $\mathrm{R}$, Irish $\mathrm{A}$. Infectious complications in indigenous renal transplant recipients in Western Australia. Intern Med J. 2017;47(6):648-55.
87. Chelluri LK, Vasantha A, Ratnakar KS. Impact of ethnicity, donor status and HLA matching on renal allograft survival: a single center study. Saudi journa of kidney diseases and transplantation : an official publication of the Saudi Center for Organ Transplantation, Saudi Arabia. 2009;20(6):995-7.

88. Kucirka LM, et al. Association of race and age with survival among patients undergoing dialysis. JAMA: Journal of the American Medical Association. 2011;306(6):620-6

89. Arce $C M$, et al. Trends in relative mortality between Hispanic and nonHispanic whites initiating dialysis: a retrospective study of the US renal data system. Am J Kidney Dis. 2013;62(2):312-21.

90. Pugh JA, Tuley MR, Basu S. Survival among MEXICAN-AMERICANS, nonHISPANIC whites, and AFRICAN-AMERICANS with end-stage renal disease the emergence of a minority pattern of increased incidence and prolonged survival. Am J Kidney Dis. 1994;23(6):803-7.

91. Murthy BVR, Molony DA, Stack AG. Survival advantage of Hispanic patients initiating dialysis in the United States is modified by race. J Am Soc Nephrol. 2005;16(3):782-90.

92. Medina RA, et al. Minority advantage in diabetic end-stage renal disease survival on hemodialysis: due to different proportions of diabetic type? Am J Kidney Dis. 1996;28(2):226-34.

93. Ríos Burrows $\mathrm{N}$, et al. Survival on Dialysis among American Indians and Alaska natives with diabetes in the United States, 1995-2010. Am J Public Health. 2014;\$3:S490-5.

94. Hall YN, et al. Differential mortality and transplantation rates among Asians and Pacific islanders with ESRD. J Am Soc Nephrol. 2005:16(12):3711-20.

95. Fernandes NMD, et al. Association of Ethnicity and Survival in peritoneal Dialysis: a cohort study of incident patients in Brazil. Am J Kidney Dis. 2013; 62(1):89-96.

96. Cole $\mathrm{N}$, et al. Black ethnicity predicts better survival on dialysis despite greater deprivation and co-morbidity: a UK study. Clin Nephrol. 2014; 82(2):77-82

97. Frankenfield DL, et al. Differences in mortality among Mexican-American, Puerto Rican, and Cuban-American dialysis patients in the United States. Am J Kidney Dis. 2009:53(4):647-57.

98. Gomez-Puerta JA, et al. Racial and ethnic differences in mortality and cardiovascular events among patients with end-stage renal disease due to lupus nephritis. Arthritis Care \& Research. 2015;67(10):1453-62.

99. Lopes AA, et al. Health-related quality of life and associated outcomes among hemodialysis patients of different ethnicities in the United States: the Dialysis outcomes and practice patterns study (DOPPS). Am J Kidney Dis. 2003:41(3):605-15.

100. Mapes $\mathrm{DL}$, et al. Health-related quality of life in the Dialysis outcomes and practice patterns study (DOPPS). Am J Kidney Dis. 2004:44(SUPPL. 2):S54-60.

101. Hicks LS, et al. Differences in health-related quality of life and treatment preferences among black and white patients with end-stage renal disease. Qual Life Res. 2004;13(6):1129-37.

102. Jain $\mathrm{P}$, et al. Survival and transplantation in end-stage renal disease: a prospective study of a multiethnic population. Nephrology Dialysis Transplantation. 2009:24(12):3840-6.

103. Tonelli $\mathrm{M}$, et al. Patient and allograft survival of indo Asian and east Asian dialysis patients treated in Canada. Kidney Int. 2007;72(4):499-504.

104. Harada. Risk Factors Associated with graft loss and patient survival after kidney Transplantation; 2009.

105. El-Sabrout R, et al. African American and Caucasian renal transplant recipients have equal acute rejection rates under sirolimus and tacrolimus combination therapy. Graft. 2002;5(8):455-61.

106. Ilori TO, et al. Racial and ethnic disparities in graft and recipient survival in elderly kidney transplant recipients. J Am Geriatr Soc. 2015;63(12):2485-93.

107. Karachristos $A$, et al. Outcomes of renal transplantation in older high risk recipients: is there an age effect? J Surg Res. 2010;161(2):173-8.

108. Douzdijian $V$, et al. Effect of race on outcome after kidney and kidneypancreas transplantation in type 1 diabetic patients. Diabetes Care. 1997; 20(8):1310-4.

109. Young BA, et al. Racial and ethnic differences in incident myocardial infarction in end-stage renal disease patients: the USRDS. Kidney Int. 2006;69(9):1691-8.

110. Abbott KC, et al. Hospitalized congestive heart failure after renal transplantation in the United States. Ann Epidemiol. 2002;12(2):115-22.

111. Joshy $\mathrm{G}$, et al. Ethnic differences in the natural progression of nephropathy among diabetes patients in New Zealand: hospital admission rate for renal complications, and incidence of end-stage renal disease and renal death. Diabetologia. 2009;52(8):1474-8. 
112. Leggat JE Jr, et al. An analysis of risk factors for withdrawal from dialysis before death. J Am Soc Nephrol. 1997;8(11):1755-63.

113. Perry $E$, et al. Peer mentoring: a culturally sensitive approach to end-of-life planning for long-term dialysis patients. Am J Kidney Dis. 2005;46(1):111-9.

114. Song M-K, et al. Racial differences in outcomes of an advance care planning intervention for dialysis patients and their surrogates. J Palliat Med. 2016; 19(2):134-42.

\section{Publisher's Note}

Springer Nature remains neutral with regard to jurisdictional claims in published maps and institutional affiliations.

Ready to submit your research? Choose BMC and benefit from:

- fast, convenient online submission

- thorough peer review by experienced researchers in your field

- rapid publication on acceptance

- support for research data, including large and complex data types

- gold Open Access which fosters wider collaboration and increased citations

- maximum visibility for your research: over $100 \mathrm{M}$ website views per year

At $\mathrm{BMC}$, research is always in progress.

Learn more biomedcentral.com/submissions 